\title{
SLEEP
}

\section{Nap time? C. elegans observed nodding off in microfluidic chambers}

Gonzales, D.L., Zhou, J. Fan, B. and Robinson, J.T. Nat Commun 10, 5035 (2019)

The worms just kept flatlining.

Daniel Gonzales, then a PhD student in Jacob Robinson's lab at Rice University in Texas, would diligently set up

Caenorhabditis elegans in the chambers of a microfluidic device they had developed that was capable of recording electrophysiology for extended periods of time, and then he would step away while the experiment ran. When he returned to plot the data, he started noticing stretches without electrical activity. Were the worms somehow slipping off the electrodes and managing to replace themselves, he wondered?

At closer look, they remained in proper place the whole time-just motionless.

The pattern repeated itself in different sized chambers, with and without electrodes. Curiosity took over, and the engineeringfocused lab took a diversion into biology. "We just decided to go knee-deep into the project to figure out what was actually happening," Gonzales says.
The worms, it turns out, were simply falling asleep.

Developing worms are known to sleep as they transition between larval stages, but the behavior is less frequent in adults-such quiescence can be triggered by stressors, but otherwise grown worms tend to remain awake. To confirm that the behavior observed in the microfluidics chambers could indeed be called 'sleep', Gonzales and his colleagues put the sleepy heads through the paces.

When other animals, including you and I, sleep, there are a few conserved criteria: decreased responsiveness to stimuli (to minimize innocuous interruptions); reversibility (we do need to be able to be woken up in the event of danger); and homeostasis (we sleep when we're tired). The worms showed all three. They slumbered on through gentle taps but could be woken by noxious blue light. When the lab kept them awake they then tended to sleep longer in response. Whole brain recordings revealed a notable state transition between being asleep and being awake, correlating the behavior with neural activity.

The results add microfluidic-induced sleep as a new means for those interested in sleep to study the behavior, says Gonzales-in their paper published in Nature Communications, they also show several ways to manipulate sleep, such as changing the worms' diet or adding thermal or mechanical stress. As the use of microfluidic devices is common in the worm field, there's also a note of caution: experimental design can dramatically increase or decrease sleep, Gonzales says. Whether or not you want your worms to nod off in their chambers is a factor that might be worth taking into account.

\section{Ellen P. Neff}

Published online: 9 December 2019

https://doi.org/10.1038/s41684-019-0450-3

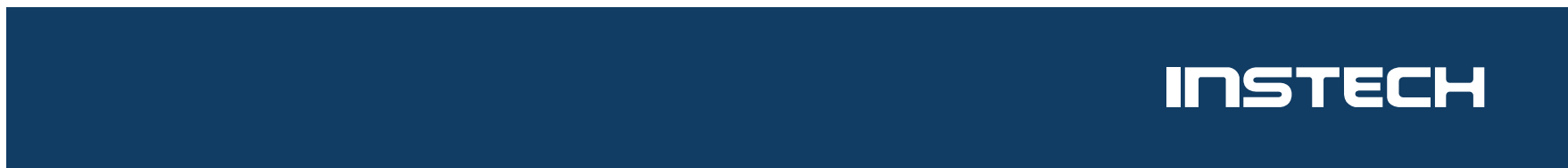

\section{Oral gavage, refined.}

Our plastic feeding needles have flexible tubes with soft tips to reduce trauma, and they're disposable to eliminate the hassle of cleaning.

Available in a range of sizes for mice, rats and other rodents. www.instechlabs.com
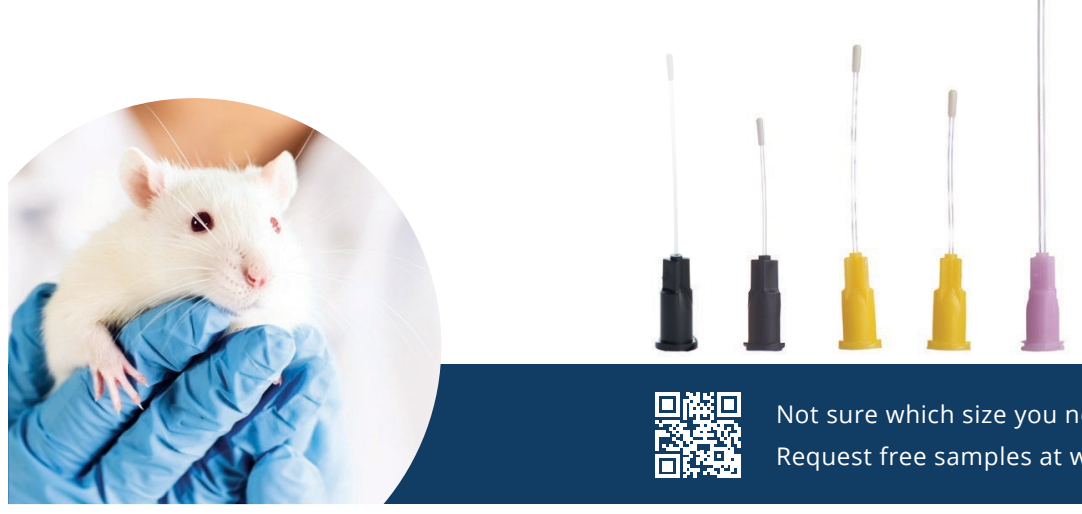